\title{
Current progresses of 3D bioprinting based tissue engineering
}

\author{
Zeyu Zhang ${ }^{1,2}$ and Xiu-Jie Wang ${ }^{1, *}$ \\ ${ }^{1}$ Key Laboratory of Genetic Network Biology, Collaborative Innovation Center of Genetics and Development, Institute of \\ Genetics and Developmental Biology, Chinese Academy of Sciences, Beijing 100101, China. \\ 2 University of Chinese Academy of Sciences, Beijing 100049, China. \\ * Correspondence: xjwang@genetics.ac.cn
}

Received October 7, 2016; Revised December 24, 2016; Accepted January 7, 2017

\begin{abstract}
Background: The shortage of available organs for transplantation is the major obstacle hindering the application of regenerative medicine, and has also become the desperate problem faced by more and more patients nowadays. The recent development and application of 3D printing technique in biological research (bioprinting) has revolutionized the tissue engineering methods, and become a promising solution for tissue regeneration.

Results: In this review, we summarize the current application of bioprinting in producing tissues and organoids, and discuss the future directions and challenges of 3D bioprinting.

Conclusions: Currently, 3D bioprinting is capable to generate patient-specialized bone, cartilage, blood vascular network, hepatic unit and other simple components/tissues, yet pure cell-based functional organs are still desired.
\end{abstract}

\section{INTRODUCTION}

With the increasing incidence of cancers and other diseases which may lead to organ dysfunction or failures, the gap between the numbers of organ donors and waiting recipients continues to enlarge. Finding effective ways to produce artificial tissues/organs is a big challenge for both basic research and regenerative medicines. One promising solution for this problem is to generate organs through tissue engineering methods, which utilize biomaterials or biomaterials in combination of cells to fabricate functional tissue/organ substitutes or tissue/organs [1]. Significant progresses have been achieved in the tissue engineering field in the past few years, such as the production of artificial bladder by mixing patientautologous bladder urothelial and muscle cells with collagen-polyglycolic acid [2], and the repopulation of cardiomyocytes on decellularized mouse heart to regain beating capacity [3]. Multiple types of biocompatible materials have also been developed, which could mimic extracellular matrix (ECM) to support cell growth $[4,5]$. However, till now, the tissue engineering method could only produce simple tissues or organoids with limited bioactivities and functions, generation of cell-based functional organs still remains as a big challenge.
The recent broad application of three-dimensional printing (3D printing) technology has demonstrated its value in clinical medication: $3 \mathrm{D}$ printed patient-specific organ models have helped clinicians to rehearse or better design surgeries [6,7], and some 3D printed biomaterials have been implanted into patients to facilitate selfregeneration of impaired tissue/organ [8,9]. Moreover, $3 \mathrm{D}$ printing offers a feasible way to manufacture bioartificial organs stereoscopically, which could in theory be able to produce organs with in vivo-like biofunctions. Here we review the recent progresses and applications of $3 \mathrm{D}$ printing in tissue engineering, the so called bioprinting. In addition to the current application on simple organs or organoids, such as bone, cartilage, blood vessels and hepatic unit, it is expected that pure-cell based functional organs could be produced by bioprinting in the near future.

\section{BASIC CONCEPT AND PROCEDURE OF 3D BIOPRINTING}

Three-dimensional bioprinting refers to the process of producing cell-based tissues/organs using 3D printing technologies. Ideally, the viability and functions of cells should be preserved in the printed tissues/organs [10]. A 
general workflow of 3D bioprinting consists of five main steps: imaging, printing strategy design, bioink design, bioprinting, and post-bioprinting processes including object maturation and assays (Figure 1) [5]. With the help of computed tomography (CT) or magnetic resonance imaging (MRI) and computer-aided design (CAD), the shape and structure of a patient's organ can be converted into a digitalized model to guide the path of 3D bioprinting. Currently, two printing strategies are commonly applied on tissue engineering: (i) to print biomaterials into scaffolds for cell seeding; or (ii) to directly print the mixture of biomaterials and cells. When selecting the printing strategies, the natural composition of the objective organs should be taken into consideration. For instance, organs with a large proportion of ECM may be more convenient to be printed by the first strategy.

Materials used in 3D bioprinting (bioink) could be classified as non-cell biomaterials and cells $[11,12]$. The non-cell biomaterials could be further grouped as natural and synthesized biomaterials (Table 1) [33,34]. The natural biomaterials refer to substances existing in living organisms, such as collagen and fibronectin [35,36], whereas the synthesized biomaterials include polycaprolactone (PCL), polyethylene glycol (PEG) and others $[37,38]$. The synthesized materials could be further modified by adding chemical groups to acquire certain physical and/or chemical properties, for example, adding of diacrylate to PEG produces PEGDA (polyethylene glycol diacrylate), which could be crosslinked under visible light. So far, various types of somatic cells or stem cells have been applied in 3D bioprinting (Table 2), but each has its own pros and cons. Somatic cells may possess desired biological functions, but could be more vulnerable during the printing process, and be difficult to re-establish cell-to-cell connections after printing. It is also uneasy to obtain all types of differentiated cells of an organ to serve as the bio-ink for printing. Pluripotent stem cells have the potential to differentiate into multiple cell types, therefore would be less constrained by the cell differentiation obstacles $[37,42]$. However, establishing proper conditions to induce post-printing differentiation could be very challenging, and the post-printing differentiation process may introduce heterogeneity and tumor risk.

Proper printer selection is one of the key issues of 3D bioprinting. The current widely-used 3D printers for bioprinting include inkjet [43], microextrusion [44] and laser-assisted bioprinters $[5,45]$. As the ultimate goal of $3 \mathrm{D}$ bioprinting is to produce tissues/organs with biofunctions, the printing speed and printing pressure should be adjusted to ensure the viability of cells during and after the printing process. For example, when using microextrusion 3D printer, low printing pressure with low printing speed will prolong the printing period, thus may influence the activity of cells, however, high printing pressure with fast printing speed may damage cells and affect the viability and biofunctions of printed organs. Once being printed out, the target organ should be in vitro/ vivo cultured to mature and tested for cell survival rate and biological functions. Validated organs can be further applied in basic research, drug screen or regenerative medicine.

\section{EXAMPLES OF BIOPRINTED TISSUES}

\section{Bone and cartilage}

Fabrication of bone-like tissue is a relative simple process comparing to complex organs. The commonly used

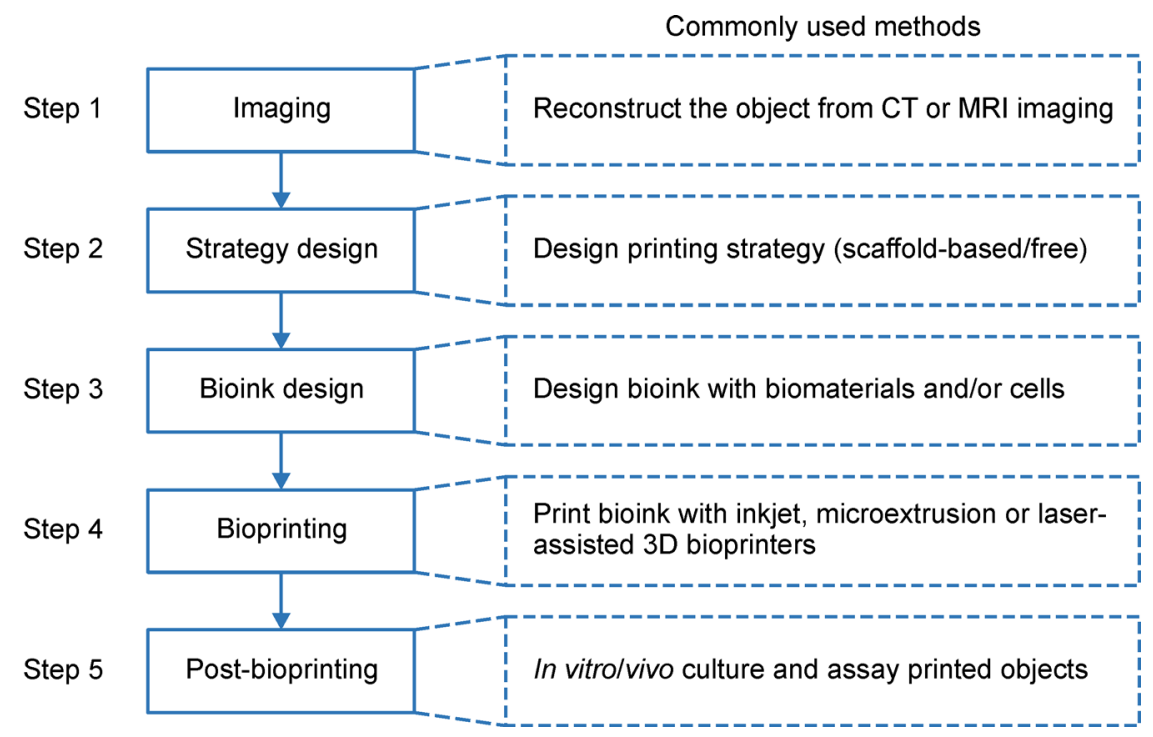

Figure 1. A common workflow of bioprinting. 
Table 1. Summary of the commonly used natural and synthetic materials in bioprinting.

\begin{tabular}{|c|c|c|c|c|c|c|c|}
\hline & & Bone & Cartilage & Ear & Skin & Hepatic unit & Tubular structured organoid \\
\hline \multirow{10}{*}{$\begin{array}{l}\text { Natural } \\
\text { materials }\end{array}$} & Alginate & & {$[13]$} & {$[14]$} & & {$[15,16]$} & {$[15]$} \\
\hline & Collagen & & [13] & & [17-21] & & \\
\hline & Fibrinogen & & [13] & & [21] & [15] & {$[15,22-24]$} \\
\hline & Gelatin & & & & & {$[15,25]$} & {$[15,23,24,26]$} \\
\hline & ECM & & & {$[27]$} & & & \\
\hline & Agarose & & & & & & [28] \\
\hline & Dextran & [29] & & & & & [28] \\
\hline & Glucose & & & & & & [28] \\
\hline & Sucrose & & & & & & [28] \\
\hline & Chitosan & & & & & [25] & \\
\hline \multirow{3}{*}{$\begin{array}{l}\text { Synthetic } \\
\text { materials }\end{array}$} & PCL & {$[30]$} & {$[13,31]$} & {$[27]$} & & & \\
\hline & PEG & & [32] & {$[27]$} & & & \\
\hline & HA & {$[4,5,29]$} & & & & & \\
\hline
\end{tabular}

Abbreviations: ECM, extracellular matrix; PCL, polycaprolactone; PEG, polyethylene glycol; HA, hydroxyapatite.

Table 2. Summary of commonly used cell types in bioprinting.

\begin{tabular}{|c|c|c|c|c|c|c|}
\hline & Bone & Cartilage & Ear & Skin & $\begin{array}{l}\text { Hepatic } \\
\text { unit }\end{array}$ & Tubular structured organoid \\
\hline Pluripotent stem cell & & & {$[27]$} & {$[21]$} & {$[16,39]$} & {$[15]$} \\
\hline Chondrocyte & & {$[13,32]$} & {$[14]$} & & & \\
\hline Endothelial cell & & & & & & {$[22,23,25,26,28,40,41]$} \\
\hline Fibroblast & & & & {$[17-20]$} & & {$[23,26,40]$} \\
\hline Hepatocyte & & & & & {$[15,25,28]$} & \\
\hline Keratinocyte & & & & {$[17-20]$} & & \\
\hline Mesenchymal stem sell & {$[4,29,30]$} & & {$[27]$} & {$[21]$} & & [23] \\
\hline Smooth muscle cell & & & & & & [40] \\
\hline Epithelial cell & & & & & & [24] \\
\hline
\end{tabular}

strategy for bone-like tissue is to print weight-loading scaffold with either cells or osteogenic molecules (Figure 2A) [46]. Cells could help to compensate bone loss whereas osteogenic molecules are able to activate selfosteogenesis. In general, the bone-shaped scaffold is produced by nano-hydroxyapatite (nHA) or PCL [47], complemented with mesenchymal stem cells (MSC), or bone morphogenetic protein (BMP) to assist the formation of mineralized tissue [13,29,30,32]. A study to compare the effects of cells and osteogenic molecules in bone regeneration has shown that, by replacing the native load-bearing bone of sheep with PCL scaffolds carrying either MSC or BMP-7, the scaffold with BMP-7 generated more bone tissue than that with MCS and the control after 3 months, implying the promising therapeutic use of such strategy in bone replacement [32].

Cartilage is another tissue which has been successfully engineered by 3D bioprinting with relatively simple processes [46]. As cartilage is less hard and rigid than bones, it is usually made from PEG and PCL with the addition of chondrocyte [33]. In a pilot study, Tao Xu and colleagues [31] fabricated a well-organized cartilage by cross-stacking of the supportive layers (made from PCL, pluronic F-127 and acetone) and functional layers (made from chondrocyte, fibrinogen and collagen; Figure 2B). After 2 weeks of in vitro culture and 8 weeks of in vivo culture, the fabricated cartilage showed fine deposition of collagen and glycosaminoglycan, resembling the organization and mechanical properties of natural cartilages. Xiaofeng Cui [27] and colleagues had applied a different strategy by using a natural osteochodral plug (extracted from bovine femoral condyles) as scaffold instead of synthesized polymers, then printed human chondrocytes mixed with modified PEG (polyethylene glycol dimethacrylate) onto the plug using thermal inkjet printer (Figure 2C), and were able to generate integrated and stabilized new cartilage tissue after 6 weeks of culture. In another trial, Chang H. Lee and colleagues replaced the sheep native meniscus with a PCL-printed scaffold carrying connective tissue growth factor (CTGF) and transforming 


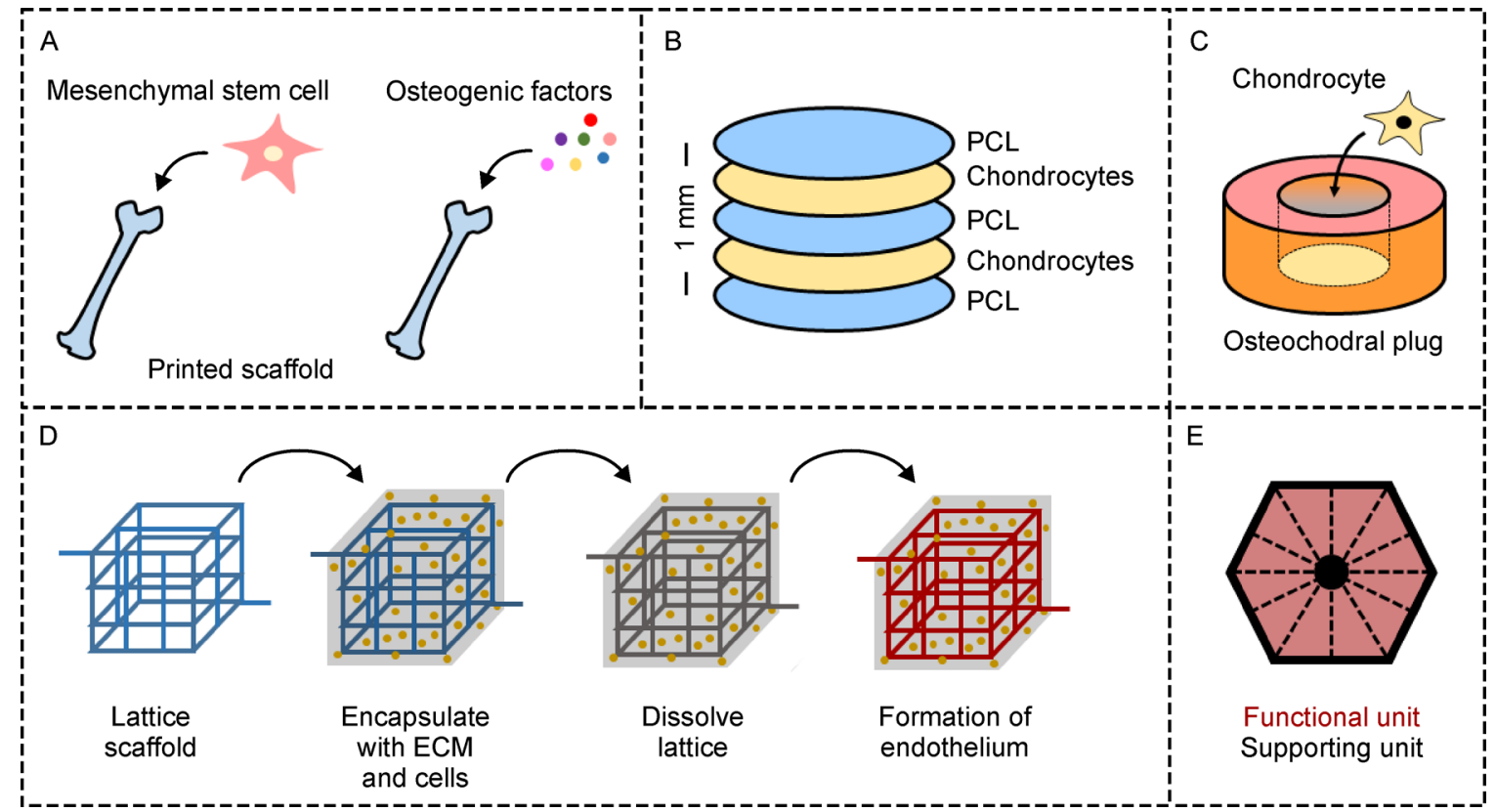

Figure 2. Examples of tissues/organoids generated by bioprinting. (A) Two typical strategies for bone fabrication. (B) The stacking method to fabricate cartilage; PCL, polycaprolactone. (C) Direct printing of chondrocytes into bovine osteochodral plug. (D) The fabrication workflow of a perfusable vascular network; ECM, extracellular matrix; (E) The organization of a printed hepatic unit: the functional units contain hepatic progenitor cells derived from human induced pluripotent stem cells, and the supporting units contain human umbilical vein endothelial cells and adipose-derived stem cells.

growth factor- $\beta 3$ (TGF- $\beta$ ). These factors stimulated endogenous cells to reconstruct ECM (collagen) and enabled sheep to resume walking capacity about 12 weeks after surgery [14].

Researchers had also fabricated cartilage-enriched bionic outer ears with similar strategies and materials as mentioned above. Printed ear-shaped structure had been produced by hydrogel (alginate, atelocollagen and decellularized ECM) encapsulating human adiposederived stem cells (ASCs) and human MSCs with the mechanical assistance of sacrificial layers, which were made from the mixture of PEG and PCL yet being removed after printing [12]. The artificial ear nicely resembled the appearance of real ears, yet its biological functions still need to be tested. In another study, Manu S. Mannoor and colleagues [17] printed chondrocytes and alginate hydrogel into ear shape, which embedded a coiled antenna with electrode. The fabricated ear had high cell viability after printing ( $\sim 91.3 \%)$ and was capable of transmitting sound signals with the help of the antenna. However, how to integrate such artificial ear into human auditory system remains challenging.

\section{Skin}

The fabrication of soft tissues, such as skin, does not require strong mechanical support, thus, hydrogels, usually collagen, are commonly used materials for this purpose [18]. Hydrogels could to a large extent resemble the natural ECM, and had been successfully applied in 3D bioprinting to produce fabricated skin with fibroblasts and keratinocytes [19-21], as well as mesenchymal stem cells [48]. Bio-ink for artificial skin (mixture of cells and hydrogel) could also be directly printed onto the wounded area of mouse model by laser-assisted printers [20,48], and had demonstrated faster wound healing ability than the control $[20,48]$.

\section{EXAMPLES OF 3D BIOPRINTED ORGA- NOIDS}

\section{Tubular structured organoids}

Mammalian organs have elegant microstructure filled with various cell types and complex blood vascular network. Hence fabricating a functional organ requires precise design and control of the printing procedure, appropriate design of bio-ink, and most importantly, the construction of functional blood vascular network to support cell growth $[40,49,50]$. Sufficient blood supply is the pre-requisite for manufacturing large organs. Natural materials such as fibrinogen have been widely used to synthesize blood vascular network, and endothelial cells (sometimes smooth muscle cells and fibroblasts) could be 
lined to the inner walls of the synthesized vascular network to mimic the endothelium of blood vessels $[22,26,41]$. In a pilot work to engineering vascular networks by printing fibrin channels with simultaneous depositing of human microvascular endothelial cells into the inner layer of the fibrin channels, cells showed proliferation at day 7 post-printing, however, by day 14, the channels started to leak thus could no longer serve as blood vessels [28].

Tubular structure can also be obtained by embedding channels into hydrogel. By this method, materials that can be easily dissolved or liquefied are firstly printed into segment, network or lattice, and then encapsulated by hydrogel. Upon perfusion, materials inside the gel bulk will be removed hence to form vascular structure. Applying such strategy, different groups had fabricated various vascularized tissue models by incorporating somatic cells into hydrogel and endothelial cells into the perfusion solutions $[23,26]$. For instance, Jordan S. Miller and colleagues [25] had incorporated 10T1/2 cells, HEK293T cells, and hepatocytes into three independent hydrogel bulks (Figure 2D), and demonstrated that the vascular product could support nutrient supply and angiogenesis partially resembling the functions of real blood vessels. Besides adding endothelial cells into the perfusion solutions, Kimberly A. Homan and colleagues [24] had perfused epithelial cells into the printed tubular lumen, which then made a 3D convoluted renal proximal tubule. Such 3D renal tubule model showed both morphological and functional enhancements as compared to the $2 \mathrm{D}$ cultured cell models.

\section{Hepatic unit}

To date, no cell-based functional liver has been fabricated. Instead, liver analogues are usually made from either hepatocytes [15,51] or stem cells [16,39], as well as natural materials such as alginate and gelatin. The functions of engineered liver analogues are usually examined by the expression of liver-specific genes and the metabolic ability of certain chemicals [39]. Seung Hyun Ahn and colleagues [16] printed preosteoblast cells, human ASCs and alginate into a mesh structure, which was further cultured using hepatogenic medium, and the expressions of liver-specific genes ( $A L B$ and TDO2) were detected after 27 days. In another study, Xuanyi Ma and colleagues [39] had fabricated liver units with a hepatic lobule-like hexagonal structure (Figure 2E) by printing the mixture of gelatin methacrylate (GelMA) and human induced pluripotent stem cell-derived hepatic progenitor cells (hiPSC-HPCs) as functional units, together with the mixture of glycidal methacrylate-hyaluronic acid, GelMA, human umbilical vein endothelial cells and human ASCs as the supporting units. Such printed liver units could better resemble the function of native hepatic lobules than that of hepatocytes cultured under the traditional 2D system [39].

\section{FUTURE PERSPECTIVES}

Although significant progresses in tissue engineering have been achieved with the application of 3D bioprinting, further efforts are still needed to improve the biological functions of the currently available bioprinting products and to fabricate more complicated organs. To achieve these goals, changes in both bio-printers and printing conditions are expected to better adapt the biological features of cells and organs, especially to provide proper culture and nutrient conditions to support cell survival and maintain cell functions during the printing process. The incorporation of blood vascular networks should be a must for generating complex organs, and close collaboration between scientists from the biological and engineering field is also essential.

Besides improvements of the biological functions and complexity of printed tissues/organs, how to manufacture human-scale tissues/organs through $3 \mathrm{D}$ bioprinting is also a big challenge. First, the large amount of cells needed for printing human-scale tissues/organs is both labor and cost intensive, and is hard to popularize under the current conditions. Second, technical obstacles of both the printing methods and culturing conditions remain to be solved to maintain the viability of printed human-scale tissues/organs. Third, restoring proper functions of printed tissues/organs is extremely difficult, which requires the proper arrangement of printed cell types, cell directions, cell connections, blood vessels as well as neural fibers, etc. Fourth, ethic issues need to be taken into account and proper regulatory policies on the manufacture and application of printed human-scale tissues/organs need to be implemented.

Despite of these challenges, 3D bioprinting is still a promising and feasible solution for regenerative medicine. We expect many more functional tissues and organs being produced by 3D bioprinting in the near future, which may serve as new systems for fundamental biological research, drug effect test and personalized drug screen. Most importantly, the production of functional tissues and organs will have profound impact on medical treatment; we hope those ideal days will come soon.

\section{ACKNOWLEDGEMENTS}

This work is supported by grants 31271349 from the National Natural Science Foundation of China, China 973 Program 2014CB964901 and CAS Strategic Priority Research Program grant XDA01020105. 


\section{COMPLIANCE WITH ETHICS GUIDELINES}

The authors Zeyu Zhang and Xiu-Jie Wang declare they have no conflict of interest.

This article does not contain any studies with human or animal subjects performed by any of the authors.

\section{REFERENCES}

1. Langer, R. and Vacanti, J. P. (1993) Tissue engineering. Science, 260, 920-926

2. Atala, A., Bauer, S. B., Soker, S., Yoo, J. J. and Retik, A. B. (2006) Tissue-engineered autologous bladders for patients needing cystoplasty. Lancet, 367, 1241-1246

3. Lu, T.-Y., Lin, B., Kim, J., Sullivan, M., Tobita, K., Salama, G. and Yang, L. (2013) Repopulation of decellularized mouse heart with human induced pluripotent stem cell-derived cardiovascular progenitor cells. Nat. Commun., 4, 2307

4. Pati, F., Jang, J., Ha, D. H., Won Kim, S., Rhie, J. W., Shim, J. H., Kim, D. H. and Cho, D. W. (2014) Printing three-dimensional tissue analogues with decellularized extracellular matrix bioink. Nat. Commun., 5, 3935

5. Murphy, S. V. and Atala, A. (2014) 3D bioprinting of tissues and organs. Nat. Biotechnol., 32, 773-785

6. Giannopoulos, A. A., Mitsouras, D., Yoo, S. J., Liu, P. P., Chatzizisis, Y. S. and Rybicki, F. J. (2016) Applications of 3D printing in cardiovascular diseases. Nat. Rev. Cardiol., 13, 701-718

7. Powers, M. K., Lee, B. R. and Silberstein, J. (2016) Threedimensional printing of surgical anatomy. Curr. Opin. Urol., 26, 283-288

8. Zopf, D. A., Hollister, S. J., Nelson, M. E., Ohye, R. G. and Green, G. E. (2013) Bioresorbable airway splint created with a threedimensional printer. N. Engl. J. Med., 368, 2043-2045

9. Xiao, Z., Tang, F., Tang, J., Yang, H., Zhao, Y., Chen, B., Han, S., Wang, N., Li, X., Cheng, S., et al. (2016) One-year clinical study of NeuroRegen scaffold implantation following scar resection in complete chronic spinal cord injury patients. Sci. China Life Sci., 59, 647-655

10. Dababneh, A. B. and Ozbolat, I. T. (2014) Bioprinting technology: a current state-of-the-art review. J. Manuf. Sci. Eng., 136, 061016

11. Hölzl, K., Lin, S., Tytgat, L., Van Vlierberghe, S., Gu, L. and Ovsianikov, A. (2016) Bioink properties before, during and after 3D bioprinting. Biofabrication, 8, 032002

12. Jose, R. R., Rodriguez, M. J., Dixon, T. A., Omenetto, F. and Kaplan, D. L. (2016) Evolution of bioinks and additive manufacturing technologies for 3D bioprinting. ACS Biomater. Sci. Eng., 2, 1662-1678

13. Fricain, J. C., Schlaubitz, S., Le Visage, C., Arnault, I., Derkaoui, S. M., Siadous, R., Catros, S., Lalande, C., Bareille, R., Renard, M., et al. (2013) A nano-hydroxyapatite - pullulan/dextran polysaccharide composite macroporous material for bone tissue engineering. Biomaterials, 34, 2947-2959

14. Lee, C. H., Rodeo, S. A., Fortier, L. A., Lu, C., Erisken, C. and Mao, J. J. (2014) Protein-releasing polymeric scaffolds induce fibrochondrocytic differentiation of endogenous cells for knee meniscus regeneration in sheep. Sci. Transl. Med., 6, 266ra171

15. Yan, Y., Wang, X., Pan, Y., Liu, H., Cheng, J., Xiong, Z., Lin, F., Wu, R., Zhang, R. and Lu, Q. (2005) Fabrication of viable tissueengineered constructs with $3 \mathrm{D}$ cell-assembly technique. Biomaterials, 26, 5864-5871

16. Ahn, S. H., Lee, H. J., Lee, J. S., Yoon, H., Chun, W. and Kim, G. H. (2015) A novel cell-printing method and its application to hepatogenic differentiation of human adipose stem cell-embedded mesh structures. Sci. Rep., 5, 13427

17. Mannoor, M. S., Jiang, Z., James, T., Kong, Y. L., Malatesta, K. A., Soboyejo, W. O., Verma, N., Gracias, D. H. and McAlpine, M. C. (2013) 3D printed bionic ears. Nano Lett., 13, 2634-2639

18. Ng, W. L., Wang, S., Yeong, W. Y. and Naing, M. W. (2016) Skin bioprinting: impending reality or fantasy? Trends Biotechnol., 34, 689-699

19. Koch, L., Deiwick, A., Schlie, S., Michael, S., Gruene, M., Coger, V., Zychlinski, D., Schambach, A., Reimers, K., Vogt, P. M., et al. (2012) Skin tissue generation by laser cell printing. Biotechnol. Bioeng., 109, 1855-1863

20. Michael, S., Sorg, H., Peck, C. T., Koch, L., Deiwick, A., Chichkov, B., Vogt, P. M. and Reimers, K. (2013) Tissue engineered skin substitutes created by laser-assisted bioprinting form skin-like structures in the dorsal skin fold chamber in mice. PLoS One, 8, e57741

21. Lee, V., Singh, G., Trasatti, J. P., Bjornsson, C., Xu, X., Tran, T. N., Yoo, S. S., Dai, G. and Karande, P. (2014) Design and fabrication of human skin by three-dimensional bioprinting. Tissue Eng. Part C Methods, 20, 473-484

22. Norotte, C., Marga, F. S., Niklason, L. E. and Forgacs, G. (2009) Scaffold-free vascular tissue engineering using bioprinting. Biomaterials, 30, 5910-5917

23. Kolesky, D. B., Homan, K. A., Skylar-Scott, M. A. and Lewis, J. A. (2016) Three-dimensional bioprinting of thick vascularized tissues. Proc. Natl. Acad. Sci. USA, 113, 3179-3184

24. Homan, K. A., Kolesky, D. B., Skylar-Scott, M. A., Herrmann, J., Obuobi, H., Moisan, A. and Lewis, J. A. (2016) Bioprinting of 3D convoluted renal proximal tubules on perfusable chips. Sci. Rep., 6,34845

25. Miller, J. S., Stevens, K. R., Yang, M. T., Baker, B. M., Nguyen, D. H., Cohen, D. M., Toro, E., Chen, A. A., Galie, P. A., Yu, X., et al. (2012) Rapid casting of patterned vascular networks for perfusable engineered three-dimensional tissues. Nat. Mater., 11, 768-774

26. Kolesky, D. B., Truby, R. L., Gladman, A. S., Busbee, T. A., Homan, K. A. and Lewis, J. A. (2014) 3D bioprinting of vascularized, heterogeneous cell-laden tissue constructs. Adv. Mater. Weinheim, 26, 3124-3130

27. Cui, X., Breitenkamp, K., Finn, M. G., Lotz, M. and D’Lima, D. D. (2012) Direct human cartilage repair using three-dimensional bioprinting technology. Tissue Eng. Part A, 18, 1304-1312

28. Cui, X. and Boland, T. (2009) Human microvasculature fabrication using thermal inkjet printing technology. Biomaterials, 30, 62216227

29. Wang, H., Li, Y., Zuo, Y., Li, J., Ma, S. and Cheng, L. (2007) 
Biocompatibility and osteogenesis of biomimetic nano-hydroxyapatite/polyamide composite scaffolds for bone tissue engineering. Biomaterials, 28, 3338-3348

30. Keriquel, V., Guillemot, F., Arnault, I., Guillotin, B., Miraux, S., Amédée, J., Fricain, J. C. and Catros, S. (2010) In vivo bioprinting for computer- and robotic-assisted medical intervention: preliminary study in mice. Biofabrication, 2, 014101

31. Xu, T., Binder, K. W., Albanna, M. Z., Dice, D., Zhao, W., Yoo, J. J. and Atala, A. (2013) Hybrid printing of mechanically and biologically improved constructs for cartilage tissue engineering applications. Biofabrication, 5, 015001

32. Reichert, J. C., Cipitria, A., Epari, D. R., Saifzadeh, S., Krishnakanth, P., Berner, A., Woodruff, M. A., Schell, H., Mehta, M., Schuetz, M. A., et al. (2012) A tissue engineering solution for segmental defect regeneration in load-bearing long bones. Sci. Transl. Med., 4, 141ra93

33. Sadtler, K., Singh, A., Wolf, M. T., Wang, X., Pardoll, D. M. and Elisseeff, J. H. (2016) Design, clinical translation and immunological response of biomaterials in regenerative medicine. Nat. Rev. Mater., 1, 16040

34. Chia, H. N. and Wu, B. M. (2015) Recent advances in 3D printing of biomaterials. J. Biol. Eng., 9, 4

35. Caliari, S. R. and Burdick, J. A. (2016) A practical guide to hydrogels for cell culture. Nat. Methods, 13, 405-414

36. Pereira, R. F. and Bártolo, P. J. (2015) 3D bioprinting of photocrosslinkable hydrogel constructs. J. Appl. Polym. Sci., 132, 42458

37. Lei, M. and Wang, X. (2016) Biodegradable polymers and stem cells for bioprinting. Molecules, 21, 539

38. Zhu, W., Ma, X., Gou, M., Mei, D., Zhang, K. and Chen, S. (2016) $3 \mathrm{D}$ printing of functional biomaterials for tissue engineering. Curr. Opin. Biotechnol., 40, 103-112

39. Ma, X., Qu, X., Zhu, W., Li, Y.-S., Yuan, S., Zhang, H., Liu, J., Wang, P., Lai, C. S. E., Zanella, F., et al. (2016) Deterministically patterned biomimetic human iPSC-derived hepatic model via rapid 3D bioprinting. Proc. Natl. Acad. Sci. USA, 113, 2206-2211

40. Kang, H. W., Lee, S. J., Ko, I. K., Kengla, C., Yoo, J. J. and Atala,
A. (2016) A 3D bioprinting system to produce human-scale tissue constructs with structural integrity. Nat. Biotechnol., 34, 312-319

41. Hoch, E., Tovar, G. E. M. and Borchers, K. (2014) Bioprinting of artificial blood vessels: current approaches towards a demanding goal. Eur. J. Cardiothorac. Surg., 46, 767-778

42. Irvine, S. A. and Venkatraman, S. S. (2016) Bioprinting and Differentiation of Stem Cells. Molecules, 21, 1188

43. Saunders, R. E. and Derby, B. (2014) Inkjet printing biomaterials for tissue engineering: bioprinting. Int. Mater. Rev., 59, 430-448

44. Zhao, Y., Li, Y., Mao, S., Sun, W. and Yao, R. (2015) The influence of printing parameters on cell survival rate and printability in microextrusion-based 3D cell printing technology. Biofabrication, 7, 045002

45. Devillard, R., Pagès, E., Correa, M. M., Kériquel, V., Rémy, M., Kalisky, J., Ali, M., Guillotin, B. and Guillemot, F. (2014). Chapter 9 - Cell Patterning by Laser-Assisted Bioprinting. In Methods in Cell Biology, Matthieu, P. and Manuel, T., eds. 159-174. New York: Academic Press

46. Wang, X., Ao, Q., Tian, X., Fan, J., Wei, Y., Hou, W., Tong, H. and Bai, S. (2016) 3D bioprinting technologies for hard tissue and organ engineering. Materials (Basel), 9, 802

47. Yi, H., Ur Rehman, F., Zhao, C., Liu, B. and He, N. (2016) Recent advances in nano scaffolds for bone repair. Bone Res., 4, 16050

48. Skardal, A., Mack, D., Kapetanovic, E., Atala, A., Jackson, J. D., Yoo, J. and Soker, S. (2012) Bioprinted amniotic fluid-derived stem cells accelerate healing of large skin wounds. Stem Cells Transl. Med., 1, 792-802

49. Kim, J. J., Hou, L. and Huang, N. F. (2016) Vascularization of three-dimensional engineered tissues for regenerative medicine applications. Acta Biomater., 41, 17-26

50. Mandrycky, C., Wang, Z., Kim, K. and Kim, D.-H. (2016) 3D bioprinting for engineering complex tissues. Biotechnol. Adv., 34, 422-434

51. Li, S., Xiong, Z., Wang, X., Yan, Y., Liu, H. and Zhang, R. (2009) Direct fabrication of a hybrid cell/hydrogel construct by a doublenozzle assembling technology. J. Bioact. Compat. Polym., 24, 249-265 